\title{
FETAL FOOT LENGTH AND HAND LENGTH: RELATIONSHIP WITH CROWN RUMP LENGTH AND GESTATIONAL AGE
}

\author{
Garima Sharma ${ }^{1}$, Aribam Jaishree Devi², Thounaojam Naranbabu Singh ${ }^{3}$
}

${ }_{1}^{1}$ Post Graduate Trainee, Department of Anatomy, Regional Institute of Medical Sciences, Imphal, Manipur.

${ }^{2}$ Associate Professor, Department of Anatomy, Regional Institute of Medical Sciences, Imphal, Manipur.

${ }^{3}$ Professor, Department of Anatomy, Regional Institute of Medical Sciences, Imphal, Manipur.

\section{ABSTRACT}

\section{BACKGROUND}

Estimation of gestational age of fetus is of great medicolegal importance. Multiple parameters of the fetal anatomical measurements are in use. However, gestational age assessment may be difficult in fetus with anencephaly, hydrocephalus, short limb dysplasia, post mortem destruction or in mutilated case. Study of literature suggests that fetal foot has a characteristic pattern of normal growth and the fetal foot shows gradual increase in length relative to the length of the embryo and could be used to estimate gestational age. The purpose of the present study is to determine the accuracy in estimating gestational age using fetal foot and hand length by studying its relation with crown rump length in the foetuses of Manipuri origin.

\section{AIMS AND OBJECTIVES}

1) To study the relationship between fetal crown rump length and fetal hand and foot length, thereby determining the accuracy in estimating gestational age by a cross-sectional study.

\section{MATERIALS AND METHODS}

A total of 100 formalin fixed fetuses of Manipuri origin, obtained from the Department of Obstetrics and Gynaecology, Regional Institute of Medical Sciences, Imphal, were included in the study, carried out in the Department of Anatomy, from February 2015 to July 2015. The parameters studied were crown rump length, foot length and hand length of fetuses. The data was analysed using SPSS software by regression analysis. Graphs were also plotted to determine pattern of growth and their correlation with crown rump length if any.

\section{RESULTS}

A total of 100 fetuses were studied, of which 43 were females and 57 were males. The mean foot length and hand length progressively increased with increase in crown rump length. Measurements were not significantly different in right or left side or among male and female fetuses. A statistically significant linear relationship was seen between foot length and crown rump length of the fetus ( $r=0.980, p<0.0001)$ and hand length and crown rump length of the fetus $(r=0.986, p<0.0001)$.

\section{CONCLUSION}

In the present study, fetal hand and foot lengths have been found to highly correlate with crown rump length and thereby gestational age. Therefore, these parameters could be utilized to estimate gestational age.

\section{KEYWORDS}

Fetal Foot Length, Hand Length, Crown Rump Length, Gestational Age.

HOW TO CITE THIS ARTICLE: Garima Sharma, Aribam Jaishree Devi, Thounaojam Naranbabu Singh. "Fetal Foot Length and Hand Length: Relationship with Crown Rump Length and Gestational Age." Journal of Evolution of Medical and Dental Sciences 2015; Vol. 4, Issue 102, December 21; Page: 16786-16792, DOI: 10.14260/jemds/2015/2517

\section{INTRODUCTION}

Estimation of gestational age of fetus is of great medicolegal importance. ${ }^{1}$ The fundamentals of the perinatal care include accurate assessment of gestational age and evaluation of the fetal growth. Multiple parameters of the fetal anatomical measurements are in use for the assessment of gestation by ultrasound like fetal Crown to Rump Length (CRL), Biparietal

Financial or Other, Competing Interest: None.

Submission 01-12-2015, Peer Review 02-12-2015,

Acceptance 15-12-2015, Published 21-12-2015.

Corresponding Author:

Garima Sharma,

Post Graduate Trainee,

Department of Anatomy,

Regional Institute of Medical Sciences,

Imphal-795004, Manipur, India.

E-mail:drgsolan@gmail.com

DOI:10.14260/jemds/2015/2517
Diameter (BPD), Head Circumference (HC), Abdominal Circumference (AC), Femur Length (FL), foot length and appearance of fetal heel ossification centers.

Physical parameters like crown heel length and weight of fetus and by noting morphological features, organ development and appearance of ossification centers can also be used. All these measurements give an accurate assessment of the fetal gestational age. However, gestational age assessment may be difficult in fetus with anencephaly, hydrocephalus, short limb dysplasia, post mortem destruction or in mutilated case.2,3

Study of literature suggests that fetal foot has a characteristic pattern of normal growth and the fetal foot shows gradual increase in length relative to the length of the embryo and could be used to estimate gestational age.4,5

Crown rump length has already been established as a highly valuable and trustworthy parameter in fetal age 
estimation by showing a significant correlation with gestational age..$^{4,6,7}$

It is also a non-invasive method to calculate gestational age, simple to carry on without any prior special training. It is also less time consuming and more economical.

The purpose of the present study is to determine the accuracy in estimating gestational age using fetal foot and hand length by studying its relation with crown rump length, as it is a simple and non-invasive technique in the fetuses of Manipuri origin.

\section{MATERIALS AND METHODS}

The current study was carried out in the Department of Anatomy, Regional Institute of Medical Sciences, Imphal from February 2015 to July 2015.

A total of 100 formalin fixed fetuses obtained from the Department of Obstetrics and Gynaecology with the permission of concerned authority and parents were included in the study.

Institutional ethics committee has no objection on doing research work on these fetuses.

Embryos less than 10cm Crown Rump Length (CRL) and fetus with gross malformation were excluded from the study.

The parameters studied were crown rump length, foot length and hand length of fetus. The measurements were recorded on both sides, right and left and in centimeters (cms). The measurements were done using a sliding vernier callipers to the nearest of millimetre.

Fetal foot length was measured in the plantar and longitudinal plane from the posterior heel to the tip of longest toe and hand length was taken on palmar surface in longitudinal plane from wrist crease to the tip of the middle finger. (Figure 1-2).

To avoid interobserver bias, the measurements were carried out by only one of the investigators. All the measurements were done three times and the mean value was used in analysis. The data was analysed using SPSS software by regression analysis. Graphs were also plotted to determine pattern of growth and their correlation with crown rump length if any.

\section{RESULTS}

A total of 100 fetus were studied, of which 43 were females and 57 were males.

Minimum of $10 \mathrm{cms}$ CRL and a maximum of $36 \mathrm{cms}$, CRL was recorded. Maximum number of fetus had 14 and $16 \mathrm{cms}$ CRL ( 9 each) followed by $13 \mathrm{cms}$ CRL (7).

Maximum number of male fetus were seen with $16 \mathrm{cms}$ CRL (7) followed by $13 \mathrm{cms}$ CRL (6). No male fetus was seen in $12 \mathrm{cms}, 33 \mathrm{cms}$ and $35 \mathrm{cms}$ CRL.

Maximum number of female fetus were seen with $14 \mathrm{cms}$, $23 \mathrm{cms}, 25 \mathrm{cms}$ and $32 \mathrm{cms}$ CRL (4 each). No female fetus was seen in $11 \mathrm{cms}, 15 \mathrm{cms}, 18 \mathrm{cms}, 27 \mathrm{cms}$ and $36 \mathrm{cms}$ CRL. Overall, for both sexes no fetus was studied with $12 \mathrm{cms}$ CRL.

The mean foot length and hand length progressively increased with increase in crown rump length and measurements were not significantly different in right or left side or among male and female fetuses.

The mean foot length with male and female distribution has been shown in Table 1 .
The mean hand length with male and female distribution has been shown in Table 2 .

Statistical analysis of the fetal foot and hand length with crown rump length has been shown in Tables 3-8 and Figure 3-4.

A statistically significant linear relationship was seen between foot length and crown rump length of the fetus $(r=0.980, p<0.0001)$ and hand length and crown rump length of the fetus $(r=0.986, p<0.0001)$.

\section{DISCUSSION}

Streeter first evaluated the fetal foot for gestational age assessment in 1920.4 Hern's then elaborated a strong relationship between fetal foot and gestational age. ${ }^{8}$

The period of gestation estimated by measurement of fetal foot length appears to be in aggrement with other ultrasound parameters. ${ }^{9,10}$

Patil SS et al. have demonstrated a statistically significant relationship between crown rump length and gestational age. 11

This study provides a normative data on fetal foot and hand growth throughout gestation. A statistically significant linear relationship is seen between foot length $(r=0.980$, $\mathrm{p}<0.0001)$ and hand length with crown rump length $(\mathrm{r}=0.986$, $\mathrm{p}<0.0001$ ). This is in accordance to work done by Patil SS et al,Bardale R et al, Arshad $\mathrm{M}$ et al, Joshi K S et al, Hebbar S et al., Platt LD et al., Mital $\mathrm{M}$ et al. and Manjunatha $\mathrm{B}$ et al.2,3,6,10,12,13,14,15 (Table 9-12).

As reported by Streeter, there is gradual increase in the length of foot relative to the length of the embryo.

The difference of this study with other studies can be explained by variations in socioeconomic status, environmental and nutritional factors with reference to the findings of other studies.

\section{CONCLUSION}

In the present study, fetal hand and foot lengths have been found to highly correlate with crown rump length, thereby gestational age and therefore these parameters could be utilized to estimate gestational age. Usage of foot and hand length can serve as an adjunct data when other parameters of fetus like crown rump length, weight, etc. cannot be utilized either due to a disease, deformity or when fragmented specimens of fetus are available in forensic and pathological studies. Of all the other parameters used to assess the gestational age, this appears to be equally accurate. These measurements can also be used as a parameter during clinical assessment as well as in USG, in premature babies, babies too ill for other measurements or in case of babies receiving incubator or intensive care.

The use of foot length and hand length in measurement of gestational age needs to be used more frequently in day to day working, so that the technique can be popularized more as its accuracy has already been reported by many workers.

\section{ACKNOWLEDGEMENT}

We acknowledge constant cooperation and help of the faculty and staff of Department of Anatomy, Regional Institute of Medical Sciences, Imphal, during the study period. 


\section{REFERENCES}

1. Castellena C, Kosa F. Morphology of the cervical vertebrae in the fetal-neonatal human skeleton. J Anat 1999;194:147-52.

2. Arshad M, Ghaus F, Nasir N, et al. Determination of Gestational Age by Measurements of Hand-A Morphometric Study in Human Foetuses. Shrinkala 2014 May;1(9):1-4.

3. Mittal M, Gupta P, Nanda V. Fetal Gestational Age Estimation by Fetal Foot Length Measurement and Fetal Femur to Foot Length Ratio in Indian Popuation-A Prospective Study. JEMDS 2014 March;3(10):2620-25.

4. Streeter GL. Weight, Sitting Height, Head Size, Foot Length and Menstrual Age of the Human Embryo. Contrib embryo Carnegie Inst 1920;11:147-70.

5. Kumar GP, Kumar UK. Estimation of gestational age from hand and foot length. Med Sci Law 1993;33:48-50.

6. Patil SS, Wasnik RN, Deokar RB, et al. Foot Length and Hand Length: Most Reliable Parameters to Estimate the Gestational Age. Medico-Legal Update 2014 JanuaryJune;14(1):144-50.

7. Sadler TW. Langman's medical embryology. $11^{\text {th }}$ ed. Philadelphia:Lippincott Williams and Wilkins; 2010.

8. Hern MW. Correlation of Fetal Age and Measurements Between 10 and 26 Weeks of Gestation. Obstetrics and Gynaecology 1984 January;63(1):26-32.

9. Mercer BM, Sklar S, Shariatmader A, et al. Fetal foot length as a predictor of gestational age. Am J Obstet Gynaecol 1987; 156:350-55.

10. Hebbar S, Kopal S, Adiga P, et al. Fetal foot length throughout gestation: a normogram. Sri Lanka Journal of Obstetrics \& Gynaecology 2013 June:58-61.

11. Patil SS, Wasnik RN, Deokar RB. Estimation of gestational age using crown heel length and crown rump length in India. International J of Healthcare \& Biomedical Research 2013 Oct;2(1):12-20.

12. Bardale R, Sonar V. Assessment of Gestational Age from Hand \& Foot Length. Indian Journal of Forensic Medicine \& Pathology 2008;1(2):47-51.

13. Joshi KS, Marahatta SB, Karki S, et al. Fetal Foot Length and Femur/Foot Length Ratio : Significance in Nepalese Context. NJR 2011;1(1):15-22.
14. Platt LD, Medaris AL, De Vore GR, Horenstein JM, Carlson DE, Bear HS. Fetal Foot Length : Relationship to Menstrual Age and Fetal Measurements in the Second Trimester. Obstet Gynecol 1998;71(4):526-31.

15. Manjunatha B, Nithin MD, Sameer S. Cross-sectional study to determine gestational age by metrical measurements of foot length. Egypt J Forensic Sci 2012;2:11-17.

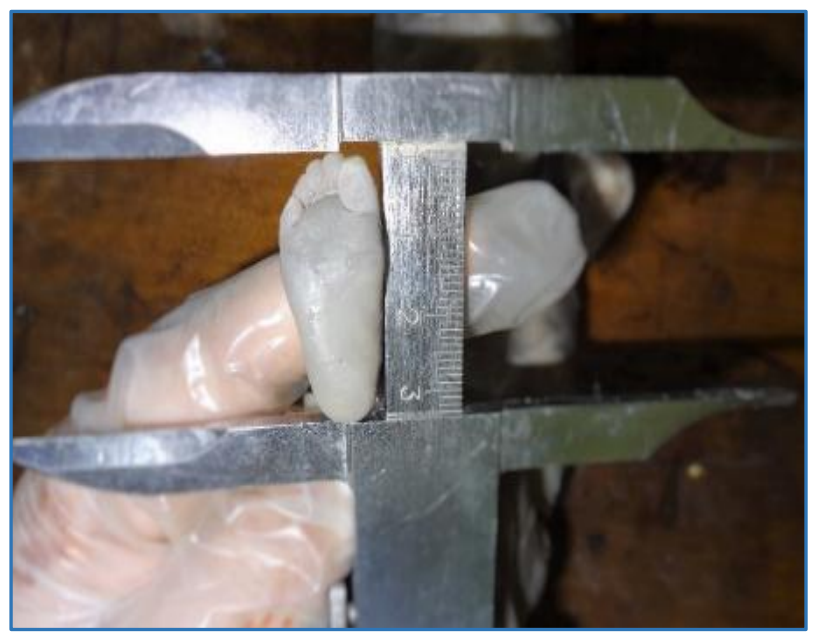

Fig. 1: Measurement of Fetal Foot Length

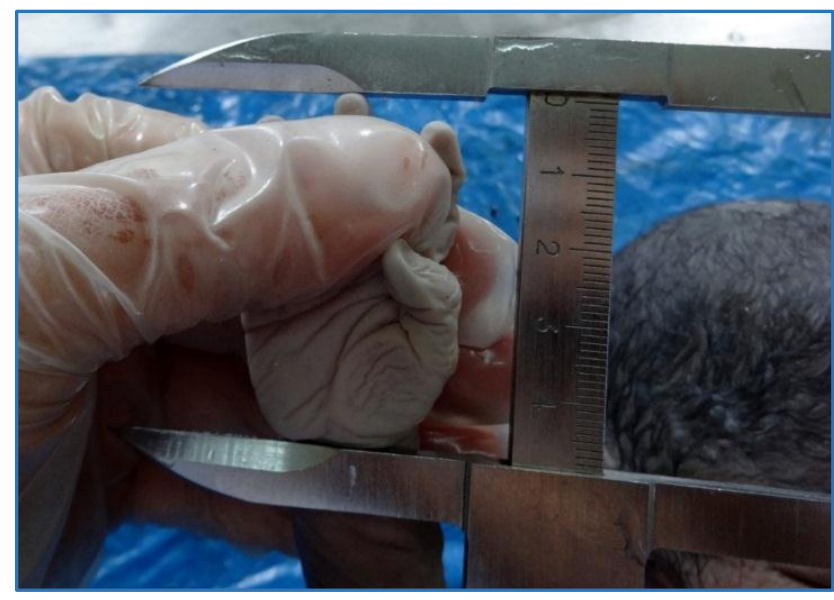

Fig. 2: Measurement of Fetal Hand Length 


\begin{tabular}{|c|c|c|c|c|c|c|}
\hline \multirow{2}{*}{$\begin{array}{c}\text { CRL } \\
\text { (cms) }\end{array}$} & \multicolumn{2}{|r|}{ MALES } & \multicolumn{2}{|r|}{ FEMALES } & \multirow[b]{2}{*}{ TOTAL } & \multirow{2}{*}{$\begin{array}{l}\text { MEAN FOOT } \\
\text { LENGTH } \\
\text { (cms) }\end{array}$} \\
\hline & $\mathbf{N}$ & $\begin{array}{l}\text { FOOT LENGTH } \\
\text { (MEAN) }\end{array}$ & $\mathbf{N}$ & $\begin{array}{l}\text { FOOT LENGTH } \\
\text { (MEAN) }\end{array}$ & & \\
\hline 10 & 2 & 1.50 & 1 & 1.50 & 3 & 1.50 \\
\hline 11 & 4 & 1.90 & & & 4 & 1.90 \\
\hline 12 & & & & & - & - \\
\hline 13 & 6 & 2.10 & 1 & 2.10 & 7 & 2.10 \\
\hline 14 & 5 & 2.50 & 4 & 2.50 & 9 & 2.50 \\
\hline 15 & 1 & 3.00 & & & 1 & 3.00 \\
\hline 16 & 7 & 3.10 & 2 & 3.10 & 9 & 3.10 \\
\hline 17 & 1 & 3.20 & 2 & 3.20 & 3 & 3.20 \\
\hline 18 & 1 & 3.40 & & & 1 & 3.40 \\
\hline 19 & 3 & 3.80 & 1 & 3.70 & 4 & 3.75 \\
\hline 20 & 2 & 4.00 & 1 & 4.00 & 3 & 4.00 \\
\hline 21 & 1 & 4.10 & 2 & 4.10 & 3 & 4.10 \\
\hline 22 & 3 & 4.30 & 2 & 4.20 & 5 & 4.25 \\
\hline 23 & 1 & 4.50 & 4 & 4.50 & 5 & 4.50 \\
\hline 24 & 2 & 5.20 & 3 & 5.20 & 5 & 5.20 \\
\hline 25 & 2 & 5.30 & 4 & 5.20 & 6 & 5.25 \\
\hline 26 & 2 & 5.60 & 1 & 5.50 & 3 & 5.55 \\
\hline 27 & 2 & 5.90 & & & 2 & 5.90 \\
\hline 28 & 2 & 6.10 & 2 & 6.10 & 4 & 6.10 \\
\hline 29 & 1 & 6.20 & 1 & 6.20 & 2 & 6.20 \\
\hline 30 & 1 & 6.70 & 3 & 6.40 & 4 & 6.55 \\
\hline 31 & 1 & 6.90 & 1 & 6.70 & 2 & 6.80 \\
\hline 32 & 1 & 7.10 & 4 & 7.00 & 5 & 7.05 \\
\hline 33 & & & 1 & 7.10 & 1 & 7.10 \\
\hline 34 & 1 & 7.20 & 1 & 7.20 & 2 & 7.20 \\
\hline 35 & & & 3 & 7.50 & 3 & 7.50 \\
\hline 36 & 4 & 7.60 & & & 4 & 7.60 \\
\hline$T a$ & & $\begin{array}{r}\text { ses Distri } \\
\text { and Sex }\end{array}$ & Ace & $\begin{array}{l}\text { ding to CR } \\
\text { ses }(N-n u t\end{array}$ & $\begin{array}{l}\text { Foot L } \\
\text { fetus) }\end{array}$ & (cms) \\
\hline
\end{tabular}

\begin{tabular}{|c|c|c|c|c|c|c|}
\hline \multirow[b]{2}{*}{$\begin{array}{c}\text { CRL } \\
\text { (cms) }\end{array}$} & \multicolumn{2}{|r|}{ MALES } & \multicolumn{2}{|r|}{ FEMALES } & \multirow[b]{2}{*}{ TOTAL } & \multirow{2}{*}{$\begin{array}{c}\text { MEAN HAND } \\
\text { LENGTH } \\
\text { (cms) }\end{array}$} \\
\hline & $\mathbf{N}$ & $\begin{array}{l}\text { HAND LENGTH } \\
\text { (MEAN) }\end{array}$ & $\mathbf{N}$ & $\begin{array}{l}\text { HAND LENGTH } \\
\text { (MEAN) }\end{array}$ & & \\
\hline 10 & 2 & 1.20 & 1 & 1.20 & 3 & 1.20 \\
\hline 11 & 4 & 1.40 & & & 4 & 1.40 \\
\hline 12 & & & & & - & - \\
\hline 13 & 6 & 1.50 & 1 & 1.40 & 7 & 1.45 \\
\hline 14 & 5 & 1.60 & 4 & 1.60 & 9 & 1.60 \\
\hline 15 & 1 & 2.10 & & & 1 & 2.10 \\
\hline 16 & 7 & 2.20 & 2 & 2.00 & 9 & 2.10 \\
\hline 17 & 1 & 2.30 & 2 & 2.20 & 3 & 2.25 \\
\hline 18 & 1 & 2.40 & & & 1 & 2.40 \\
\hline 19 & 3 & 2.60 & 1 & 2.40 & 4 & 2.50 \\
\hline 20 & 2 & 3.10 & 1 & 3.10 & 3 & 3.10 \\
\hline 21 & 1 & 3.30 & 2 & 3.30 & 3 & 3.30 \\
\hline 22 & 3 & 3.50 & 2 & 3.40 & 5 & 3.45 \\
\hline 23 & 1 & 3.70 & 4 & 3.60 & 5 & 3.65 \\
\hline 24 & 2 & 3.90 & 3 & 3.70 & 5 & 3.80 \\
\hline 25 & 2 & 4.10 & 4 & 4.00 & 6 & 4.05 \\
\hline 26 & 2 & 4.30 & 1 & 4.20 & 3 & 4.25 \\
\hline 27 & 2 & 4.50 & & & 2 & 4.50 \\
\hline 28 & 2 & 4.60 & 2 & 4.50 & 4 & 4.55 \\
\hline 29 & 1 & 5.00 & 1 & 4.90 & 2 & 4.95 \\
\hline 30 & 1 & 5.20 & 3 & 5.00 & 4 & 5.10 \\
\hline 31 & 1 & 5.40 & 1 & 5.20 & 2 & 5.30 \\
\hline 32 & 1 & 5.60 & 4 & 5.30 & 5 & 5.45 \\
\hline 33 & & & 1 & 5.50 & 1 & 5.50 \\
\hline 34 & 1 & 5.90 & 1 & 5.70 & 2 & 5.80 \\
\hline 35 & & & 3 & 6.00 & 3 & 6.00 \\
\hline 36 & 4 & 6.30 & & & 4 & 6.30 \\
\hline \multicolumn{7}{|c|}{$\begin{array}{l}\text { Table 2: Cases Distributed According to CRL (cms), Hand Length } \\
\text { (cms) and Sex of } 100 \text { Fetuses (N - number of fetus) }\end{array}$} \\
\hline
\end{tabular}




\begin{tabular}{|c|c|c|c|c|c|c|c|c|}
\hline & \multirow{2}{*}{ Model } & \multicolumn{2}{|c|}{$\begin{array}{c}\text { Unstandardized } \\
\text { Coefficients }\end{array}$} & \multirow{2}{*}{$\begin{array}{c}\begin{array}{c}\text { Standardized } \\
\text { Coefficients }\end{array} \\
\text { Beta }\end{array}$} & \multirow{2}{*}{$\mathbf{t}$} & \multirow{2}{*}{ Sig. } & \multicolumn{2}{|c|}{$\begin{array}{c}95.0 \% \\
\text { Confidence Interval for B } \\
\end{array}$} \\
\hline & & B & Std. Error & & & & $\begin{array}{l}\text { Lower } \\
\text { Bound }\end{array}$ & $\begin{array}{l}\text { Upper } \\
\text { Bound }\end{array}$ \\
\hline \multirow{2}{*}{1} & (Constant) & -1.245 & .249 & & -4.995 & .000 & -1.759 & -.732 \\
\hline & CRL & .256 & .010 & .980 & 24.923 & .000 & .235 & .277 \\
\hline
\end{tabular}

\begin{tabular}{|c|c|c|c|c|c|c|c|c|c|}
\hline \multirow[b]{2}{*}{ Model } & \multirow[b]{2}{*}{$\mathbf{R}$} & \multirow{2}{*}{$\begin{array}{c}\mathbf{R} \\
\text { Square }\end{array}$} & \multirow{2}{*}{$\begin{array}{l}\text { Adjusted R } \\
\text { Square }\end{array}$} & \multirow{2}{*}{$\begin{array}{l}\text { Std. Error of } \\
\text { the Estimate }\end{array}$} & \multicolumn{5}{|c|}{ Change Statistics } \\
\hline & & & & & $\begin{array}{c}\text { R Square } \\
\text { Change }\end{array}$ & $\begin{array}{c}\mathbf{F} \\
\text { Change }\end{array}$ & df1 & df2 & $\begin{array}{c}\text { Sig. F } \\
\text { Change }\end{array}$ \\
\hline $\begin{array}{c}1 \\
\text { (Constant) } \\
\text { CRL }\end{array}$ & $.980^{\mathrm{a}}$ & .961 & .960 & .41558 & .961 & 621.172 & 1 & 25 & .000 \\
\hline \multicolumn{10}{|c|}{ a. Predictors: (Constant), CRL } \\
\hline
\end{tabular}

\begin{tabular}{|c|c|c|c|}
\hline \multicolumn{2}{|c|}{} & FL & CRL \\
\hline \multirow{2}{*}{ Pearson Correlation } & FL & 1.000 & .980 \\
\cline { 2 - 4 } & CRL & .980 & 1.000 \\
\hline \multirow{2}{*}{ Sig. (1-tailed) } & FL &. & .000 \\
\cline { 2 - 4 } & CRL & .000 & 27 \\
\hline \multirow{2}{*}{ N } & FL & 27 & 27 \\
\cline { 2 - 4 } & CRL & 27 & 27 \\
\hline \multicolumn{2}{|c|}{ Table 5: Correlations, Foot Length (FI) and CRL } \\
\hline
\end{tabular}

\begin{tabular}{|c|c|c|c|c|c|c|c|c|}
\hline & \multirow{2}{*}{ Model } & \multicolumn{2}{|c|}{ Unstandardized Coefficients } & \multirow{2}{*}{$\begin{array}{c}\begin{array}{c}\text { Standardized } \\
\text { Coefficients }\end{array} \\
\text { Beta }\end{array}$} & \multirow{2}{*}{$t$} & \multirow{2}{*}{ Sig. } & \multicolumn{2}{|c|}{$\begin{array}{l}95.0 \% \text { Confidence Interval } \\
\text { for B }\end{array}$} \\
\hline & & B & Std. Error & & & & $\begin{array}{l}\text { Lower } \\
\text { Bound }\end{array}$ & Upper Bound \\
\hline \multirow{2}{*}{1} & (Constant) & -1.319 & .174 & & -7.592 & .000 & -1.677 & -.961 \\
\hline & CRL & .212 & .007 & .986 & 29.632 & .000 & .197 & .227 \\
\hline \multicolumn{9}{|c|}{ Table 6: Coefficient of Dependable Variables of Hand Length } \\
\hline
\end{tabular}

\begin{tabular}{|c|c|c|c|c|c|c|c|c|c|}
\hline \multirow{2}{*}{ Model } & \multirow{2}{*}{$\mathbf{R}$} & \multirow{2}{*}{$\begin{array}{c}\text { R } \\
\text { Square }\end{array}$} & \multirow{2}{*}{$\begin{array}{l}\text { Adjusted R } \\
\text { Square }\end{array}$} & \multirow{2}{*}{$\begin{array}{l}\text { Std. Error of } \\
\text { the Estimate }\end{array}$} & \multicolumn{5}{|c|}{ Change Statistics } \\
\hline & & & & & $\begin{array}{c}\text { R Square } \\
\text { Change }\end{array}$ & $\begin{array}{c}\mathbf{F} \\
\text { Change }\end{array}$ & df1 & df2 & $\begin{array}{c}\text { Sig. F } \\
\text { Change }\end{array}$ \\
\hline $\begin{array}{c}\text { 1(Constant) } \\
\text { CRL }\end{array}$ & $.986^{\mathrm{a}}$ & .972 & .971 & .28959 & .972 & 878.056 & 1 & 25 & .000 \\
\hline \multicolumn{10}{|c|}{ a. Predictors: (Constant), CRL } \\
\hline
\end{tabular}

\begin{tabular}{|c|c|c|c|}
\hline \multicolumn{2}{|c|}{} & HL & CRL \\
\hline \multirow{2}{*}{ Pearson Correlation } & HL & 1.000 & .986 \\
\cline { 2 - 5 } & CRL & .986 & 1.000 \\
\hline \multirow{2}{*}{ Sig. (1-tailed) } & HL &. & .000 \\
\cline { 2 - 5 } & CRL & .000 &. \\
\hline \multirow{2}{*}{$\mathrm{N}$} & HL & 27 & 27 \\
\cline { 2 - 4 } & CRL & 27 & 27 \\
\hline
\end{tabular}

*MNFL - Mean foot length 


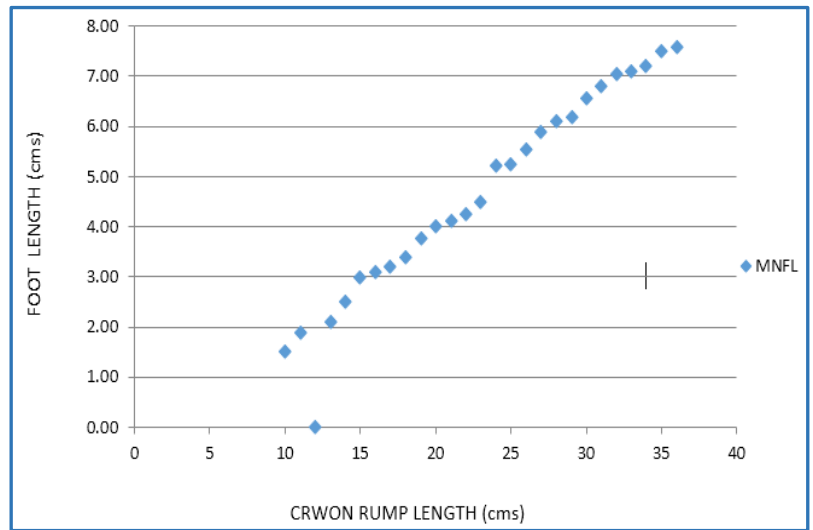

Fig.3: Scatter Diagragm of Fetal Foot Length (cms) versus CRL (cms) Demonstrating A Linear Relationship

*MNFL - Mean Foot length

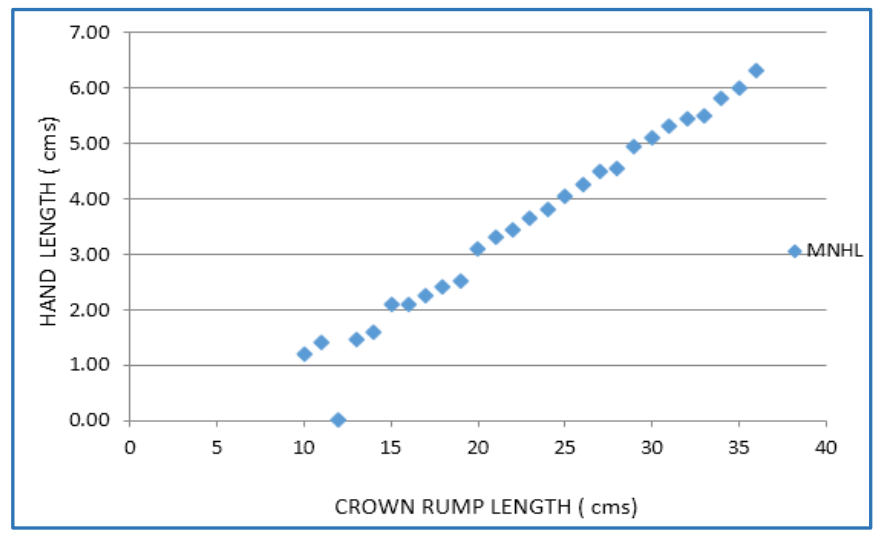

Fig. 4: Scatter Diagram of Fetal Hand Length (cms) versus CRL (cms) Demonstrating a Linear Relationship

*MNHL-Mean Hand Length

\begin{tabular}{|c|c|c|c|c|c|c|c|c|c|c|}
\hline & $\begin{array}{c}\text { PRESENT } \\
\text { STUDY }\end{array}$ & $\begin{array}{c}\text { MITTAL M } \\
\text { et al. }\end{array}$ & $\begin{array}{c}\text { KUMAR } \\
\text { GP et } \\
\text { al. }\end{array}$ & $\begin{array}{c}\text { PATIL SS } \\
\text { et al. }\end{array}$ & $\begin{array}{c}\text { MERCER BM } \\
\text { et al. }\end{array}$ & $\begin{array}{c}\text { HEBBAR S } \\
\text { et al. }\end{array}$ & $\begin{array}{c}\text { BARDALE R } \\
\text { et al. }\end{array}$ & $\begin{array}{c}\text { JOSHI } \\
\text { et al. }\end{array}$ & $\begin{array}{c}\text { PLATT } \\
\text { et al. }\end{array}$ & $\begin{array}{c}\text { MANJUNATHA B } \\
\text { et al. }\end{array}$ \\
\hline $\mathrm{r}$ & 0.980 & 0.9 & 0.97 & 0.996 & 0.981 & 0.97 & 0.975 & 0.97 & 0.94 & 0.988 \\
\hline $\mathrm{p}$ & $<0.0001$ & $<0.001$ & $<0.001$ & $<0.0001$ & & $<0.001$ & $<0.0001$ & $<0.000$ & $<0.001$ \\
\hline \multicolumn{1}{|c|}{ Table 9: Fetal Foot Length: Statistical Comparison with Other Studies } \\
\hline
\end{tabular}

\begin{tabular}{|c|c|c|c|c|c|c|c|}
\hline \multicolumn{8}{|c|}{ Foot Length } \\
\hline $\begin{array}{c}\text { CRL } \\
\text { (cms) }\end{array}$ & $\begin{array}{l}\text { PRESENT } \\
\text { STUDY }\end{array}$ & $\begin{array}{c}\text { STREETER } \\
\text { GL }\end{array}$ & $\begin{array}{l}\text { MITTAL M et } \\
\text { al. }\end{array}$ & $\begin{array}{l}\text { PATIL SS et } \\
\text { al. }\end{array}$ & $\begin{array}{l}\text { HERN MW } \\
\text { et al. }\end{array}$ & $\begin{array}{c}\text { MERCER BM } \\
\text { et al. }\end{array}$ & $\begin{array}{l}\text { HEBBAR S et } \\
\text { al. }\end{array}$ \\
\hline 10 & 1.5 & & & 1.4 & 1.4 & & \\
\hline 11 & 1.9 & 1.4 & & 1.7 & 1.7 & & \\
\hline 12 & - & & & 2.03 & 2.1 & & \\
\hline 13 & 2.1 & & & & & & \\
\hline 14 & 2.5 & 2 & 2 & & 2.2 & 2.1 & 2.63 \\
\hline 15 & 3 & & 2.5 & 2.6 & 2.4 & & \\
\hline 16 & 3.1 & & 2.7 & & 2.6 & & \\
\hline 17 & 3.2 & & 3.1 & 3.025 & 2.9 & & \\
\hline 18 & 3.4 & & & 3.45 & & & \\
\hline 19 & 3.75 & & & & 3.2 & 3.3 & 3.669 \\
\hline 20 & 4 & & 3.5 & 3.65 & 3.4 & & \\
\hline 21 & 4.1 & 4 & 3.8 & 4.025 & 3.7 & & \\
\hline 22 & 4.25 & & 4.2 & 4.275 & 3.8 & & \\
\hline 23 & 4.5 & & & 4.5 & 3.9 & 4.4 & 4.569 \\
\hline 24 & 5.2 & & & 4.89 & 4.1 & & \\
\hline 25 & 5.25 & & 4.9 & 5.2 & & & \\
\hline 26 & 5.55 & & & 4.455 & & & \\
\hline 27 & 5.9 & & 5.4 & 5.75 & & 5.8 & 5.675 \\
\hline 28 & 6.1 & & & 6.02 & & & \\
\hline 29 & 6.2 & 6 & 5.8 & 6.225 & & & \\
\hline 30 & 6.55 & & 6.3 & 6.515 & & 6.3 & \\
\hline 31 & 6.8 & & 6.6 & 6.9 & & & \\
\hline 32 & 7.05 & & & & & & 6.864 \\
\hline 33 & 7.1 & & 6.9 & 7.15 & & & \\
\hline 34 & 7.2 & & 7.2 & 7.5 & & & 7.589 \\
\hline 35 & 7.5 & & 7.4 & 7.775 & & & \\
\hline 36 & 7.6 & & & & & 7.4 & \\
\hline \multicolumn{8}{|c|}{ Table 10: Mean Fetal Foot Length: Comparison with Other Studies } \\
\hline
\end{tabular}




\begin{tabular}{|c|c|c|c|c|}
\hline & $\begin{array}{c}\text { PRESENT } \\
\text { STUDY }\end{array}$ & $\begin{array}{c}\text { ARSHAD M } \\
\text { et al. }\end{array}$ & $\begin{array}{c}\text { PATIL SS } \\
\text { et al. }\end{array}$ & $\begin{array}{c}\text { BARDALE R } \\
\text { et al. }\end{array}$ \\
\hline $\mathrm{r}$ & 0.986 & & 0.985 & 0.978 \\
\hline $\mathrm{p}$ & $<0.0001$ & $<0.05$ & $<0.0001$ & $<0.0001$ \\
\hline \multicolumn{4}{|c|}{ Table 11: Fetal Hand Length : Statistical } \\
Comparison with Other Studies
\end{tabular}

\begin{tabular}{|c|c|c|c|c|}
\hline \multicolumn{2}{|c|}{ Gestational Age (Weeks ) } & \multicolumn{2}{c|}{ Arshad M et al. } \\
\hline$<17$ & & 1.716 & \\
\hline $17-20$ & & & 2.116 & \\
\hline $21-25$ & & & 3.183 & \\
\hline $26-30$ & & 3.95 & \\
\hline$>30$ & & 5.283 & \\
\hline \multicolumn{4}{|c|}{ Table 12(b) } \\
\hline
\end{tabular}

\begin{tabular}{|c|c|c|}
\hline \multicolumn{3}{|c|}{ Hand Length } \\
\hline CRL (cms) & Present Study & Patil SS et al. \\
\hline 10 & 1.2 & 1.05 \\
\hline 11 & 1.4 & 1.4 \\
\hline 12 & - & 1.55 \\
\hline 13 & 1.45 & \\
\hline 14 & 1.6 & \\
\hline 15 & 2.1 & 2.05 \\
\hline 16 & 2.1 & \\
\hline 17 & 2.25 & 2.44 \\
\hline 18 & 2.4 & \\
\hline 19 & 2.5 & 2.65 \\
\hline 20 & 3.1 & 3.05 \\
\hline 21 & 3.3 & 3.03 \\
\hline 22 & 3.45 & 3.53 \\
\hline 23 & 3.65 & 3.47 \\
\hline 24 & 3.8 & 3.89 \\
\hline 25 & 4.05 & 4.1 \\
\hline 26 & 4.25 & 4.49 \\
\hline 27 & 4.5 & 4.47 \\
\hline 28 & 4.55 & 4.78 \\
\hline 29 & 4.95 & 5.18 \\
\hline 30 & 5.1 & 5.22 \\
\hline 31 & 5.3 & 5.6 \\
\hline 32 & 5.45 & \\
\hline 33 & 5.5 & 6.05 \\
\hline 34 & 5.8 & 6.27 \\
\hline 35 & 6 & 6.24 \\
\hline 36 & 6.3 & \\
\hline \multicolumn{3}{|c|}{ Table 12(a) } \\
\hline
\end{tabular}

\begin{tabular}{|c|c|c|c|c|}
\hline \multicolumn{2}{|c|}{ Gestational Age (Weeks) } & \multicolumn{2}{c|}{ Bardale R et al. } \\
\hline $12-16$ & & & 1.4 & \\
\hline $17-20$ & & & 2.52 & \\
\hline $21-24$ & & & 3.31 & \\
\hline $25-28$ & & & 4.81 & \\
\hline $29-32$ & & & 4.81 & \\
\hline $33-36$ & & & 5.85 & \\
\hline $37-40$ & \multicolumn{3}{|c|}{ Table 12(c) } \\
\hline \multicolumn{3}{|c|}{} & 6.32 & \\
\hline
\end{tabular}

Table 12(a,b,c): Mean Fetal Hand Length : Comparison with Other Studies 